\title{
Screening for COPD: the gap between logic and evidence
}

\author{
Alan Kaplan ${ }^{1}$ and Mike Thomas ${ }^{2}$
}

Affiliations: ${ }^{1}$ Family Physician Airways Group of Canada, University of Toronto, Toronto, ON, Canada. ${ }^{2}$ Primary Care and Population Sciences Division, University of Southampton, Southampton, UK.

Correspondence: Alan Kaplan, 7335 Yonge Street, Thornhill, ON, L3T 2B2, Canada. E-mail: for4kidsaggmail.com

@ERSpublications

Screen smokers for COPD symptoms first, then confirm using spirometry; this may increase motivation to quit smoking http://ow.ly/u9ED308C5zn

Cite this article as: Kaplan A, Thomas M. Screening for COPD: the gap between logic and evidence. Eur Respir Rev 2017; 26: 160113 [https://doi.org/10.1183/16000617.0113-2016].

ABSTRACT Chronic obstructive pulmonary disease (COPD) is a common disease leading to further morbidity and significant mortality. The first step for any condition is to make the appropriate diagnosis, and spirometry barriers abound in practice around the world. It is tempting to undertake mass screening on all smokers to detect COPD. While this would pick up cases of COPD, results of studies of its effect on COPD end-points such as exacerbations, hospitalisations and mortality are disappointing. As such, aggressive case finding of COPD by screening for symptoms that patients may not themselves perceive is very important in primary care, with subsequent spirometry defining the diagnosis.

We also have to separate out population screening from individual patient interactions. Performing spirometry, even on a truly asymptomatic patient, may allow earlier diagnosis and modification of risk factors such as smoking (mostly) and exacerbation risk. It also recognises patients with early disease who are at high risk of comorbidities such as cardiac illness, such that appropriate treatment strategies can be implemented. Making a diagnosis, and even the fact of worrying about such a diagnosis, can affect the motivational level of the individual patient to cease smoking; all patients should of course be counselled to stop smoking. As such, consider the individual patient in front of you for unrecognised symptoms and therefore unrecognised illness, as making a diagnosis earlier can allow the institution of care, including smoking cessation, vaccination, bronchodilators and comorbidity management.

\section{Introduction}

Chronic obstructive pulmonary disease (COPD) is a condition worthy of more attention due to its prevalence in the general population, estimated to be $\sim 1 \%$ across all ages, rising steeply to $8-10 \%$ or more in individuals aged $\geqslant 40$ years [1], and the fact that it is projected to be the third leading cause of death by 2020 [2]. Growth in COPD continues due to the rapidly increasing smoking rates in developing countries in addition to the ageing population in developed countries. There is considerable overdiagnosis [3] as well as misdiagnosis [4], but perhaps the major issue is that of underdiagnosis, rates of which are substantially higher than those reported for high blood pressure, hypercholesterolaemia and other chronic diseases. Screening for an early diagnosis of COPD is feasible and different models have been suggested. COPD screening can be compared with screening programmes for hypertension [5] and hyperlipidaemia. Initiatives targeting these conditions have greatly reduced deaths from acute myocardial infarction or stroke in the USA. To date, the data have for COPD been more equivocal, and so have not resulted in the widespread implementation of similar screening for COPD in most health economies.

Received: Dec 062016 | Accepted after revision: Jan 232017

Conflict of interest: Disclosures can be found alongside this article at err.ersjournals.com

Provenance: Submitted article, peer reviewed.

Copyright CERS 2017. ERR articles are open access and distributed under the terms of the Creative Commons Attribution Non-Commercial Licence 4.0 
Smoking cessation is by far the most important secondary prevention intervention for patients found to have COPD; among other health benefits, it leads to a sustained small improvement in the level of forced expiratory volume in $1 \mathrm{~s}(\mathrm{FEV} 1)$ in smokers [6] and reduces the rate of decline to that of nonsmokers [7]. Pharmaceutical intervention is only of limited benefit in severe airway obstruction and does not affect the progression of disease to a relevant extent [8]. Other interventions stimulated by COPD diagnosis which may be of value include supported lifestyle changes such as improvements in exercise and diet, appropriate vaccinations and pulmonary rehabilitation. As COPD is a clear marker of its multiple comorbidities, aggressive management of these could prevent related negative outcomes. For instance, statins have shown an effect on all-cause mortality rates of patients with COPD [9]. These factors should all reduce both the personal and societal burden of the disease, but despite substantial effort and investment, the implementation of quality spirometry, the essential test in COPD diagnosis, is deficient because of multiple barriers [10] and limitations [11].

\section{Why screen?}

Screening is only justified if there is evidence of underdiagnosis and evidence of clinical and economic benefits from early diagnosis. There continues to be underdiagnosis of COPD for a variety of reasons. Often smokers with early disease and only mild airflow limitation have few symptoms or poor perception of their symptoms. Many will have such inactive lifestyles that they fail to appreciate the extent of their declining exercise tolerance. They often attribute symptoms such as cough, dyspnoea and sputum as a normal consequence of smoking. In addition, they are often concerned that they will receive the response "Well, then quit smoking" from their well-meaning physicians. In addition, physicians may miss some subtle early signs, such as frequent respiratory infections.

Experts suggest that we should look for early disease, but current guidelines favour targeted case finding rather than population screening.

The Global Initiative for Chronic Obstructive Lung Disease (GOLD) [12] states "A diagnosis of COPD should be considered in any patient who has dyspnoea, chronic cough or sputum production, and a history of exposure to risk factors".

The UK national strategy [13] describes "early recognition of disease ... through opportunistic and systematic case finding and better recognition of signs and symptoms by healthcare professionals especially those in primary care and by the population itself".

This is all complicated by the way COPD can be viewed in early disease. Patients may be "asymptomatic", but often have symptoms which they dismiss as being attributed to other factors such as ageing, smoking or fitness levels [14]. Studies have shown clearly that patients with undiagnosed GOLD stage 1 COPD will have signs of impaired quality of life and reduction in activities of daily living [15]. These can proceed to lifestyle changes that cause deconditioning, social isolation and subsequent effects on mood [16] and anxiety [17]. Symptoms may be nonspecific [18] (cough, sputum or chest infections), which may delay the diagnosis. Exacerbations, which clearly reduce lung function, particularly early in the disease [19], do occur in those with mild disease [20], and are often unreported [21] or may result in multiple health encounters that are labelled as being due to a respiratory tract infection and treated with antibiotics.

Loss of lung function seems to be most marked early in the disease, a further reason why making an earlier diagnosis has the potential to impact strongly. In fact, it seems that most lung function is lost in "mild" disease [22]. Therefore, to have an impact on the natural history of COPD, it is logical to look at the effects of treatment in the earlier stages [23] and "screen" for earlier disease.

There is evidence to show that having reduced FEV1 in COPD is in itself a marker of cardiovascular outcomes. A reduced FEV1 is a marker for cardiovascular mortality independent of age, sex and smoking history [24]. This would show the potential value of screening for COPD to ascertain cardiovascular risk.

\section{What is screening?}

The World Health Organization's criteria for screening [25] specify that the condition sought should be an important and treatable health problem in which the natural history is well understood and for which there is an agreed policy on whom and how to treat. There should be an acceptable and available test for diagnosis and resources to treat the condition, and an economic balance in the cost of finding a case related to the medical expenditure as a whole.

It must be recognised that there are negative aspects to screening. It is possible that issues that would never be of clinical concern might be identified. This could cause significant patient stress in dealing with a "disease" that would not have affected them, and would probably lead to increased healthcare costs. In addition, a negative screen could cause a false sense of security and allow continued "bad" behaviours, 
such as smoking, in a population. Treatments may have side-effects, which would be unfortunate if the treatment was truly not necessary.

While there are known treatments for COPD, their applicability to patients in the early stages of COPD with only mild airflow limitation is less clear. The diagnosis in this condition is solely based on spirometry, which still have limitations in addition to the numerous barriers. Unfortunately, there is a paucity of evidence that spirometry in early disease will lead to smoking cessation [26]. All smokers should be supported to quit regardless of their disease status. It is possible that efforts to make an early diagnosis will use resources that could be better suited to dealing with people with symptomatic disease [27].

Furthermore, screening must be performed in the correct populations, to adequately assess results. Screening tools are available, which cover several key factors, e.g. the COPD Population Screener Questionnaire [28], the Lung Function Questionnaire [29] and the Canada Lung Health Test [30]; however, depending on the populations being screened different variables must be considered [31], such as prenatal maternal smoking, low birthweight, postnatal tobacco exposure and childhood respiratory infections, which could further the precision of screening in primary care settings.

\section{Possible screening errors}

Current COPD guidelines, such as the GOLD workshop report, use a fixed FEV1/forced vital capacity (FVC) value $(0.70)$ to define airway obstruction, and FEV1 \% predicted to classify COPD severity. This approach leads to a significant percentage of false-positive diagnoses of mild and moderate COPD. Expressing FEV1 as a \% predicted value can introduce a bias: small people, elderly people and especially small elderly people who are in good respiratory health will be incorrectly identified as having an abnormally low FEV1. The use of the lower limit of normal for the FEV1/FVC ratio instead of a fixed value of 0.70 is be a possible step towards a more accurate evaluation of early airway obstruction. Other conditions can potentially lead to a reduced ratio, such as cystic fibrosis or other pulmonary conditions, emphasising the need for a clinical perspective in addition to spirometry [32].

Our current view of COPD as a prospective predictable worsening disease has recently been challenged by work that shows that as many as half of patients with physiological COPD undergo a rapid decline, but the other half have always had poor lung function [33].

\section{What is the evidence for screening for COPD?}

The most recent intensive review is from the US Preventive Services Task Force [34], which stated that there is no evidence to support identifying asymptomatic patients, as there are no definitive treatment benefits to doing so, and that "whole-population screening for COPD using spirometry has no net benefit". They estimated that only one exacerbation (a key driver in COPD costs) could be prevented by screening 455 adults aged 60-69 years using spirometry.

That said, is there any evidence that diagnosing COPD can improve smoking cessation rates, which is the single most important factor in preventing the progression of the illness? Most studies have been negative [35-37]; there have been a few positive trials [38-40]. In addition, despite concerns, there is no evidence that false reassurance in smokers with normal spirometry adversely dampens their motivation to quit if present.

Screening infers that if there is a positive test result, there is something we can do about it. Benefits of early diagnosis can include vaccination to prevent exacerbations, recommendation of regular exercise [41], more aggressive management of comorbidities and potentially even pharmacotherapy to reduce the rate of loss of lung function [42]. Making a firm diagnosis may allow identification of exacerbations of COPD as opposed to respiratory tract infection and encourage preventative measures to help prevent the more aggressive loss of lung function that exacerbations are known to cause [19].

Aggressive management of comorbidities is worthwhile, as the incidence of early vascular disease is increased in people with COPD compared with smokers without COPD or nonsmokers [43].

\section{What do patients think?}

Patients may not desire assessment of hitherto undiagnosed and asymptomatic disease. They may not be looking for firm diagnosis, and there are certainly many different patient types in this regard. In fact, some get upset about being, in their opinion, patronised. Patients say things like "I don't mind damaging my own body" [44]. Some qualitative work has been undertaken on smokers. A structured interview of 205 smokers motivated to quit who were found to have mild COPD in a screening study in the Netherlands and Belgium were asked their opinions on the making of a new diagnosis of COPD. Interestingly, $86 \%$ agreed that performing spirometry on heavy smokers is a good idea to raise awareness of the negative effects of smoking and that it might help prevent further disease and decrease smoking rates. 15\% felt that it interfered with their freedom of choice [45]. 


\section{What do physicians think?}

Primary care physicians are already under significant pressures of time, patient numbers and multimorbidity and have enough trouble dealing with the problems that patients present to them. They may feel that they do not need to "look for more work" or that they do not have the skills or capacity in spirometry to perform this kind of screening. Thus, it this was concluded that better local resources and compensation for extended consultation times with better understanding of COPD would be needed [46].

Other qualitative work tackles these issues head on. There is value to recognising that there are a lot of missed diagnoses and that interventions could be improve quality of life and prevent loss of lung function. However, there are concerns that patients may be frightened by a new diagnosis, by stigmatisation and by lack of treatment to change the condition, and about possible extra cost to healthcare systems. It was concluded that properly resourced and supported case finding of patients who recognise that they have symptoms attributable to COPD could show benefits [47].

\section{Can case finding work?}

Database studies have shown that patients can be identified retrospectively using database searches. In one study of 8000 newly diagnosed COPD patients, $75 \%$ had been treated recently with antibiotics, 35\% with oral steroids and $55 \%$ with $\beta$-agonists [48] in the previous 5 years. Another database trial has shown that most patients assessed had a lower respiratory consultation (86\%) and treatment (68\%) in the 5 years prior to diagnosis [49]. Simple office evaluations targeting early symptoms can be effective. A written invitation and questionnaire focused on risk factors and symptoms has been proven to be effective in an early detection programme [50]. Positive questionnaires with subsequent spirometry or the use of a pocket spirometer have also been shown to be effective [51, 52].

In another study, patients were randomised to either a targeted case-finding group or a routine care group. Eligible patients were ever-smokers aged 40-79 years without a previously recorded diagnosis of COPD. Patients in the targeted case-finding group were further randomly assigned (1:1) via their household to receive either a screening questionnaire at the general practitioner (GP) consultation (opportunistic) or a screening questionnaire at the GP consultation plus a mailed questionnaire (active). Respondents reporting relevant respiratory symptoms were invited for post-bronchodilator spirometry. The percentage of newly detected COPD cases was higher in the active case-finding than in the opportunistic case-finding group. Importantly, active case finding was more cost-effective than opportunistic case finding [53].

\section{What about the individual patient in front of you?}

There is clear evidence that population screening of truly asymptomatic smokers using spirometry to make a diagnosis of COPD is not cost effective. This kind of population screen, while not currently sanctioned, does occur daily in primary care, with patients being tested routinely for diabetes and hyperlipidaemia. The risk of developing COPD in a smoker is estimated at $~ 25 \%$ [54]. What is the pick-up rate for elevated lipids needing treatment in most of the patients who have them tested? There are no studies of dyslipidaemia screening in young adults [55], but the current US Preventive Services Task Force recommends that screening begins at age 35 years in all males and at 20 years for males and females at risk for coronary heart disease, despite the fact that they do not recommend treatment until the age of 40 years. What about the rates of screening of people who have an abnormal level of thyroid stimulating hormone who present with nonspecific fatigue? (Guidelines for thyroid screening are ambivalent in this regard [56].) In Canada, adults at low to moderate risk of diabetes are screened routinely for type 2 diabetes, yet Canadian recommendations advise no routine screening for type 2 disease [57].

In that case, why should we in respiratory healthcare apologise for considering screening for an expensive, severe, life-limiting disorder? The point may be somewhat moot, as the definition of COPD was changed in the new GOLD guidelines released in November 2016 [12]. The new definition includes symptoms as part of the diagnosis, so by definition we cannot screen for COPD if there are no symptoms. However, we can screen for airflow limitation, which changes the description, but probably not the discussion.

Most patients who claim to be asymptomatic are probably truly symptomatic upon full and holistic assessment. Patients with mild COPD have often changed their lives to prevent experiencing symptoms: they walk less, do less and have a poorer quality of life. The secret is to uncover these symptoms by asking the right questions. Asking a patient to compare their current activity to what they were doing a few years ago may provide insight.

In addition, making an early diagnosis of COPD can allow the earlier introduction of preventative medication for conditions that complicate earlier disease, such as coronary artery disease and depression. There is evidence that $\sim 1 \%$ of COPD patients develop lung cancer every year, which may be associated 
with genetic susceptibility to cigarette smoke; here is a potential opportunity to screen and reduce lung cancer deaths [58].

Smoking cessation is all about the motivation of the individual patient. If you feel that a motivation can be related to identifying a currently unrecognised condition in the patient, it behoves you to explore the issue further. So, without advocating population screening, it might still be appropriate to "screen" the patient in front of you. Don't apologise for it: money is currently being spent much more ludicrously with lesser outcomes in our healthcare systems.

\section{Conclusion}

The evidence shows that although population screening of all smokers for COPD using spirometry finds disease, it does not change outcomes cost effectively. However, physicians treat individual patients, and "screening" for unrecognised symptoms using, for example, the Canada Lung Health Test followed by spirometry for an accurate diagnosis is effective to establish an earlier diagnosis of COPD, for which treatment can have considerable impact. This happens for many other diseases in daily care; maybe we should rethink?

\section{References}

1 Halbert RJ, Natoli JL, Gano A, et al. Global burden of COPD: systematic review and meta-analysis. Eur Respir J 2006; 28: 523-532.

2 Mathers CD, Loncar D. Projections of global mortality and burden of disease from 2002 to 2030. PLoS Med 2006; 3: e422.

3 Bednarek M, Maciejewski J, Wozniak M, et al. Prevalence, severity, and underdiagnosis of COPD in the primary care setting. Thorax 2008; 63: 402-407.

4 Rabe KF, Hurd S, Anzueto A, et al. Global strategy for the diagnosis, management, and prevention of chronic obstructive pulmonary disease: GOLD executive summary. Am J Respir Crit Care Med 2007; 176: 532-555.

5 Burt VL, Whelton P, Roccella EJ, et al. Prevalence of hypertension in the US adult population: results from the Third National Health and Nutrition Examination Survey, 1988-1991. Hypertension 1995; 25: 305-313.

6 Scanlon PD, Connett JE, Waller LA, et al. Smoking cessation and lung function in mild-to-moderate chronic obstructive pulmonary disease: The Lung Health Study. Am J Respir Crit Care Med 2000; 161: 381-390.

7 Anthonisen NR, Skeans MA, Wise RA, et al. The effects of a smoking cessation intervention on 14.5-year mortality: a randomized clinical trial. Ann Intern Med 2005; 142: 233-238.

8 Decramer M, Gosselink R, Bartsch P, et al. Effect of treatments on the progression of COPD: report of a workshop held in Leuven, 11-12 March 2004. Thorax 2005; 60: 343-349.

9 Lahousse L, Loth DW, Joos GF, et al. Statins, systemic inflammation and risk of death in COPD: the Rotterdam study. Pulm Pharmacol Ther 2013; 26: 212-217.

10 Kaplan A, Levitz S. Use of spirometry in family practice in Canada; results of a nationwide survey. Eur Respir $J$ 2016; 48: Suppl. 60, PA3938.

11 Soriano JB, Zielinski J, Price D. Screening for and early detection of chronic obstructive pulmonary disease. Lancet 2009; 374: 721-732.

12 Global Initiative for Chronic Obstructive Lung Disease (GOLD). Pocket Guide to COPD Diagnosis, Management, and Prevention. http://goldcopd.org/pocket-guide-copd-diagnosis-management-prevention-2016/ Date last accessed: November 19, 2016.

13 UK Department of Health. An Outcomes Strategy for People with Chronic Obstructive Pulmonary Disease (COPD) and Asthma in England. www.gov.uk/government/publications/an-outcomes-strategy-for-people-withchronic-obstructive-pulmonary-disease-copd-and-asthma-in-england Date last accessed: November 19, 2016. Date last updated: July 18, 2011.

14 Price D, Freeman D, Cleland J, et al. Earlier diagnosis and earlier treatment of COPD in primary care. Prim Care Respir J 2011; 20: 15-22.

15 Miravatles M, Soriano JB, García-Río F, et al. Prevalence of COPD in Spain: impact of undiagnosed COPD on quality of life and daily life activities. Thorax 2009; 64: 863-868.

16 Katz P, Julian L, Omachi TA, et al. The impact of disability on depression among individuals with COPD. Chest 2010; 137: 838-845.

17 Wagena EJ, Kant I, van Amelsvoort LG, et al. Risk of depression and anxiety in employees with chronic bronchitis: the modifying effect of cigarette smoking. Psychosom Med 2004; 66: 729-734.

18 Lindberg A, Larsson LG, Rönmark E, et al. Decline in FEV1 in relation to incident chronic obstructive pulmonary disease in a cohort with respiratory symptoms. COPD 2007; 4: 5-13.

19 Donaldson GC, Seemungal TAR, Bhowmik A, et al. Relationship between exacerbation frequency and lung function decline in chronic obstructive pulmonary disease. Thorax 2002; 57: 847-852.

20 O'Reilly JF, Williams AE, Holt K, et al. Defining COPD exacerbations: impact on estimation of incidence and burden in primary care. Prim Care Respir J 2006; 15: 346-353.

21 Langsetmo L, Platt RW, Ernst P, et al. Underreporting exacerbation of chronic obstructive pulmonary disease in a longitudinal cohort. Am J Respir Crit Care Med 2008; 177: 396-401.

22 Bhatt SP, Soler X, Wang X, et al. Predictors of lung function decline in smokers in COPDGene phase 2. Am J Respir Crit Care Med 2015; 191: A2433.

23 Tantucci C, Modina D. Lung function decline in COPD. Int J Chron Obstruct Pulmon Dis 2012; 7: 95-99.

24 Sin DD, Wu L, Man SF. The relationship between reduced lung function and cardiovascular mortality: a population-based study and a systematic review of the literature. Chest 2005; 127: 1952-1959.

25 Wilson JMG, Jungner G. Principles and Practice of Screening for Disease. Geneva, World Health Organization, 1968. http://apps.who.int/iris/bitstream/10665/37650/17/WHO_PHP_34.pdf.

26 White P. Spirometric screening for COPD: wishful thinking, not evidence. Thorax 2007; 62: 742-743. 
Med 2008; 359: 1543-1554.

43 Eickhoff P, Valipour A, Kiss D, et al. Determinants of systemic vascular function in patients with stable chronic obstructive pulmonary disease. Am J Respir Crit Care Med 2008; 178: 1211-1218.

44 Bethea J, Murtagh B, Wallace SE. “I don't mind damaging my own body”. A qualitative study of the factors that motivate smokers to quit. BMC Public Health 2015; 15: 4.

45 Kotz D, Vos R, Huibers MJH. Ethical analysis of the justifiability of labelling with COPD for smoking cessation. J Med Ethics 2009; 35: 534-540.

46 Sandelowsky H, Hylander I, Krakau I, et al. Time pressured deprioritization of COPD in primary care: a qualitative study. Scand J Prim Health Care 2016; 14: 55-65.

47 Sharmeen T, Summers R, Astles C, et al. "Don't get me wrong, I think case finding is okay": exploring healthcare professionals' perspectives on target case-finding for COPD. Eur Respir J 2016; 14: Suppl. 60, PA3932.

48 Lippiett K, Gillett K, Longstaff J, et al. Identifying undiagnosed COPD through searches of UK routine primary care databases. Eur Respir J 2015; 46: Suppl. 59, PA338.

49 Jones RM, Price D, Ryan D, et al. Opportunities to diagnose chronic obstructive pulmonary disease in routine care in the UK: a retrospective study of a clinical cohort. Lancet Respir Med 2014; 2: 267-276.

50 Lyngsø AM, Backer V, Gottlieb V, et al. Early detection of COPD in primary care-the Copenhagen COPD Screening Project. BMC Public Health 2010; 10: 524

51 Franco-Marina F, Fernandez-Plata R, Torre-Bouscoulet L, et al. Efficient screening for COPD using three steps: a cross-sectional study in Mexico City. NPJ Prim Care Respir J 2014; 14: 14002.

52 Yawn B, Duvall K, Peabody J, et al. The impact of screening tools on diagnosis of chronic obstructive pulmonary disease in primary care. Am J Prev Med 2014; 47: 563-575.

53 Jordan R, Adab P, Sitch A, et al. Targeted case finding for chronic obstructive pulmonary disease versus routine practice in primary care (TargetCOPD): a cluster-randomised controlled trial. Lancet Respir Med 2016; 4: 720-730.

54 Løkke A, Lange P, Scharling H, et al. Developing COPD: a 25 year follow up study of the general population. Thorax 2006; 61: 935-939.

55 Chou R, Dana T, Blazina I, et al. Screening for dyslipidemia in younger adults: a systematic review for the U.S. Preventive Services Task Force. Ann Intern Med 2016; 165: 560-564.

56 U.S. Preventive Services Task Force. Guide to Clinical Preventive Services: Report of the U.S. Preventive Services Task Force. 2nd Edn. Washington, Office of Disease Prevention and Health Promotion, 1996; pp. 209-218.

57 Canadian Task Force on Preventive Health Care,Pottie K, Jaramillo A, et al. Recommendations on screening for type 2 diabetes in adults. CMAJ 2012; 184: 1687-1696.

58 Sekine Y, Katsura H, Koh E, et al. Early detection of COPD is important for lung cancer surveillance. Eur Respir J 2012; 39: 1230-1240. 